\title{
Improved upper critical field in bulk-form magnesium diboride by mechanical alloying with carbon
}

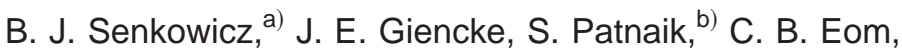 \\ E. E. Hellstrom, and D. C. Larbalestier \\ Applied Superconductivity Center and Department of Materials Science and Engineering, \\ University of Wisconsin-Madison, Madison, Wisconsin 53706
}

(Received 3 November 2004; accepted 14 March 2005; published online 11 May 2005)

\begin{abstract}
High-energy milling of magnesium diboride $\left(\mathrm{MgB}_{2}\right)$ prereacted powder renders the material largely amorphous through extreme mechanical deformation and is suitable for mechanically alloying $\mathrm{MgB}_{2}$ with dopants including carbon. Bulk samples of milled carbon and $\mathrm{MgB}_{2}$ powders subjected to hot isostatic pressing and $\mathrm{Mg}$ vapor annealing have achieved upper critical fields in excess of $32 \mathrm{~T}$ and critical current density approaching $10^{6} \mathrm{~A} / \mathrm{cm}^{2}$. (C) 2005 American Institute of Physics.

[DOI: 10.1063/1.1920428]
\end{abstract}

Recent studies of magnesium diboride thin films by Braccini et al. ${ }^{1}$ found $H_{c 2}(0 \mathrm{~K})^{\|}>50 \mathrm{~T}$ for C-doped $\mathrm{MgB}_{2}$ films. Fits to the data using the two-gap model of $H_{c 2}$ of Gurevich $^{2}$ showed an extrapolated $H_{c 2}(0 \mathrm{~K})^{\|}(H$ parallel to the $\mathrm{Mg}$ and $\mathrm{B}$ planes) of $\sim 70 \mathrm{~T}$ and $H_{c 2}(0 \mathrm{~K})^{\perp}$ exceeding $40 \mathrm{~T}^{1}$. Such critical field properties exceed those of any Nbbased conductor at any temperature, suggesting that $\mathrm{MgB}_{2}$ could be a viable replacement for $\mathrm{Nb}_{3} \mathrm{Sn}$ as a high field magnet conductor. Masui et al., ${ }^{3}$ Ohmichi et al., ${ }^{4}$ and Kazakov et $a l .{ }^{5}$ have reported carbon-doped single crystals with $H_{c 2}(0 \mathrm{~K})^{\|}$in excess of $30 \mathrm{~T}$. Untextured carbon-doped filaments fabricated by a chemical vapor deposition (CVD) method can achieve upper critical fields in excess of $30 \mathrm{~T}$ at $4.2 \mathrm{~K} .{ }^{6}$ The measured $H_{c 2}$ in this case is the higher parallel critical field $H_{c 2}^{\|}(0 \mathrm{~K})$, rather than the lower, perpendicular critical field that controls the bulk $J_{C}$. One immediate question is how thin films and bulk differ. A partial answer was given by Braccini et al. ${ }^{1}$ who noted that the $c$-axis parameter of the highest $H_{c 2}$ films was expanded relative to C-doped bulk samples, which have lower $H_{c 2}$ values.

Several groups ${ }^{7-10}$ have also produced wires containing $\mathrm{SiC}$, the latter finding that $H_{c 2}$ could attain values as high as the CVD filaments of Wilke et al. ${ }^{6}$ In fact there is increasing suspicion that one of the beneficial effects of $\mathrm{SiC}$ addition occurs by $\mathrm{C}$ doping of the $\mathrm{MgB}_{2}$. In general there is good agreement that $\mathrm{C}$ doping of $\mathrm{MgB}_{2}$ is a useful means of alloying $\mathrm{MgB}_{2}$, even if the best bulk $H_{c 2}$ values are only about half those of the best thin films. The present Letter discusses the ex situ synthesis of alloyed $\mathrm{MgB}_{2}$ powder using high energy ball milling of $\mathrm{MgB}_{2}$ with $\mathrm{C}$. Since a major goal of $\mathrm{MgB}_{2}$ technology is the fabrication of high critical current density, multifilament wire suitable for magnet applications, we need a scalable bulk process capable of producing carbon-doped precursor powder. One such method is provided by this paper.

The composition of carbon-doped $\mathrm{MgB}_{2}$ is commonly given using the format $\operatorname{Mg}\left(\mathrm{B}_{1-X} \mathrm{C}_{X}\right)_{2}$. Alfa-Aesar prereacted $\mathrm{MgB}_{2}$ powder was mixed with powdered graphite in the following proportions: $0.1 \mathrm{C}+0.9 \mathrm{MgB}_{2}$ (nominal $X=0.0525$ )

\footnotetext{
${ }^{a)}$ Electronic mail: senkowic@cae.wisc.edu

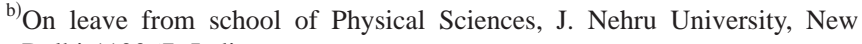
Delhi 110067, India.
}

and $0.3 \mathrm{C}+0.7 \mathrm{MgB}_{2}$ (nominal $X=0.17$ ). The mixtures were high energy ball milled in a Spex $8000 \mathrm{M}$ mixer/mill with a WC milling container and milling media. Powders were milled until no graphite peak was discerned in the $\mathrm{x}$-ray diffraction (XRD) pattern, which took $10 \mathrm{~h}$ for $X=0.0525$ and $25 \mathrm{~h}$ for $X=0.17$. The milled powders were then cold isostatic pressed to form pellets that were welded into evacuated stainless steel tubes and hot isostatic pressed (HIP) at $1000{ }^{\circ} \mathrm{C}$ and $>30 \mathrm{ksi}$ for $200 \mathrm{~min}$. These HIP-treated pellets were cut apart and examined. They were black, lusterless, and lacked mechanical strength. These features were attributed to incomplete reaction due to a deficiency of $\mathrm{Mg}$ with respect to $(\mathrm{B}+\mathrm{C})$ resulting from adding $\mathrm{C}$ to the ex situ powder without adding $\mathrm{Mg}$. Accordingly, portions of the HIP-treated pellets were welded into evacuated stainless steel tubing with a quantity of $\mathrm{Mg}$ metal that was about half the volume of the $\mathrm{MgB}_{2}$ pellet. $\mathrm{MgB}_{2}$ pellets and $\mathrm{Mg}$ metal were arranged in such a way that the sample would be exposed to $\mathrm{Mg}$ vapor, while limiting exposure to liquid $\mathrm{Mg}$, as described by Braccini et al. ${ }^{11}$ These sealed $\mathrm{MgB}_{2}+\mathrm{Mg}$ packages were heat treated at $900{ }^{\circ} \mathrm{C}$ for $5 \mathrm{~h}$ and cooled at a rate not faster than $100{ }^{\circ} \mathrm{C} / \mathrm{h}$ so as to fully react the $\mathrm{B}$ and $\mathrm{C}$ with $\mathrm{Mg}$. After cutting the samples free from the steel, the samples were found to be black in color, to gain luster when polished, and to be dense and hard. However, even after this $\mathrm{Mg}$ vapor anneal, the $\mathrm{X}$ - ray intensities were reduced and the peaks broadened as compared with unmilled powders.

Some superconducting properties were then evaluated resistively with small transport currents of $\sim 0.25 \mathrm{~A} / \mathrm{cm}^{2}$ in a 9-T Quantum Design physical properties measurement system (PPMS) and then with the 65-T short pulse magnet at the National High Magnetic Field Lab (NHMFL) in Los Alamos, NM. $H_{c 2}(T)$ was defined by the $90 \%$ normal state resistance transition $^{11}$ giving values about 5-10\% lower than would be obtained by the 95-99\% transitions. ${ }^{6}$ The hysteretic magnetization $\Delta \mathrm{M}$ of bulk samples was also measured in an Oxford Instruments vibrating sample magnetometer (VSM), from which the critical current density $J_{c}(H, T)$ was calculated assuming fully connected samples using the expression $J_{c}(H, T)=\Delta \mathrm{M} \times 12 b /\left(3 b d-d^{2}\right)$ where $b$ and $d$ are the width and thickness of a rectangular section bar.

Figure 1 compares $H_{c 2}(T)$ of our samples to those of an $X=0.035$ C filament of Wilke et al. ${ }^{6}$ Our $X=0.0525$ sample 


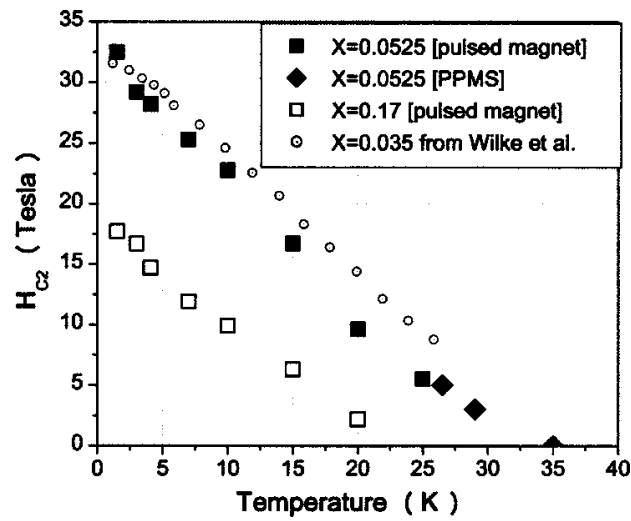

FIG. $1 . H_{c 2}$ defined by the $90 \%$ normal state resistivity of our carbon-doped samples and final onsets of full normal-state resistance for the sample of Wilke et $\mathrm{l}^{6}$

had $T_{c}$ of $35 \mathrm{~K}$ and $H_{c 2}(0 \mathrm{~K}) \sim 33 \mathrm{~T}$, and our nominal $X$ $=0.17$ sample had $T_{c}$ of $22 \mathrm{~K}$ and $\sim 19 \mathrm{~T} H_{c 2}(0 \mathrm{~K})$. The CVD filament ${ }^{6}$ exhibited very similar properties to our $X$ $=0.0525$ sample, given that $H_{c 2}(T)$ was determined from the highest fraction of the transition, rather than the $90 \%$ transition as here. ${ }^{6,12}$ These data also agree well with single crystal data for which an estimated maximum $H_{c 2}^{\|}(0 \mathrm{~K}) \sim 29 \mathrm{~T}$ at $X=0.05$ (Ref. 3) was reported.

To determine how much carbon was successfully incorporated into the lattice, Fig. 2 compares $a$-axis lattice parameters in the literature. ${ }^{5,13-15}$ Both single crystal data sets (Kazakov et al. ${ }^{5}$ and Lee et $a l_{.}{ }^{15}$ ) are rather linear in C content. Fitting the Kazakov et al. ${ }^{5}$ data produces the following relationship:

$$
a=3.08439 x-0.3153,
$$

where $x$ is the carbon content given by $\operatorname{Mg}\left(\mathrm{B}_{1-x} \mathrm{C}_{x}\right)_{2}$.

Lattice parameters determined from high resolution $\mathrm{x}$-ray diffraction measurements are presented in Table I along with the carbon content of the lattice calculated using Eq. (1). This calculation suggests that $x=0.051$ of the nominally $X=0.0525$ sample (Sample $A$ ) was incorporated into the lattice. For the nominal $X=0.17$ carbon-doped sample (Sample B), only about $40 \%$ of the available carbon was incorporated into the lattice $(x=0.069)$. Between $X=0.0525$ and $X=0.17$ we experienced diminishing efficiency of carbon incorporation, as previously observed by Bharathi et al. ${ }^{14}$

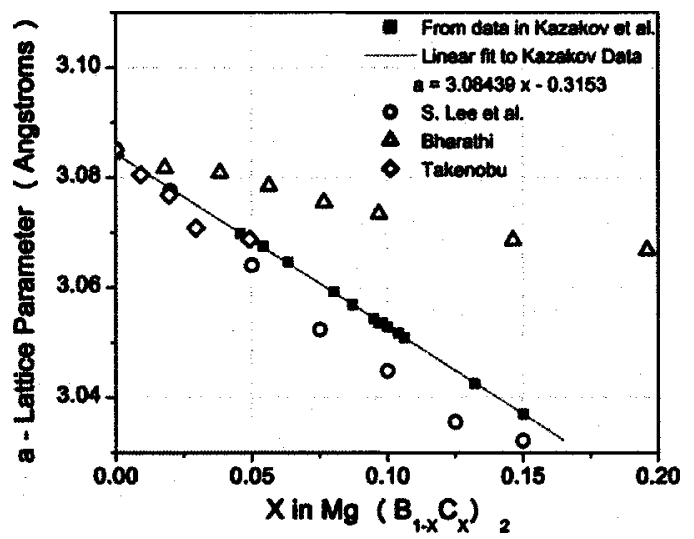

FIG. 2. $a$-axis lattice parameter as a function of carbon content. Data from

Kazakov et al. ${ }^{5}$ Takenobu $e t$ al. ${ }^{13}$ Bharathi $e t$ al. ${ }^{14}$ and Lee et al. ${ }^{15}$ zero are about half the values of $H_{c 2}(T)$ found in Fig. 1 .
Downloaded 22 Feb 2007 to 128.104 .198 .190 . Redistribution subject to AIP license or copyright, see http://apl.aip.org/a
TABLE I. Properties of C-doped milled samples.

\begin{tabular}{cccccc}
\hline \hline $\begin{array}{c}\text { Nominal } \\
X\end{array}$ & $\begin{array}{c}a \\
(\AA)\end{array}$ & $\begin{array}{c}c \\
(\AA)\end{array}$ & $\begin{array}{c}\text { Calculated } \\
X\end{array}$ & $\begin{array}{c}\rho(40 \mathrm{~K}) \\
(\mu \Omega \mathrm{cm})\end{array}$ & RRR \\
\hline 0.0525 & $3.0683(2)$ & $3.5267(5)$ & 0.051 & 960 & 1.185 \\
0.17 & $3.062(3)$ & $3.526(7)$ & 0.069 & 9000 & 1.037 \\
\hline \hline
\end{tabular}

Figure 3 shows $J_{C}(H, T)$ of the samples. $J_{C}$ of the $X$ $=0.0525$ sample had a maximum approaching $10^{6} \mathrm{~A} / \mathrm{cm}^{2}$, similar to values in the literature for HIP processed ex situ $\mathrm{MgB}_{2} .{ }^{16}$ The $X=0.17$ sample had maximum $J_{C}$ depressed two orders of magnitude, with depressed irreversibility fields $H^{*}(T)$ as well (defined as the field at which $J_{C}$ vanishes). As noted by comparing Figs. 1 and $3, H^{*}(T)$ is of order one half $H_{c 2}(T)$, for example $6 \mathrm{~T}$ at $20 \mathrm{~K}$ vs $10 \mathrm{~T}$ at $20 \mathrm{~K}$ for the $X$ $=0.051$ sample. This is consistent with $H_{c 2}(T)$ being determined by the highest $H_{c 2}(T)$ path in the samples that corresponds to $H_{c 2}^{\|}(T)$, whereas $H^{*}(T)$ is determined by the breakup of the fully connected path that starts to occur in the vicinity of $H_{c 2}^{\perp}(T)$.

As shown in Table I, the normal state resistivity values were two to three orders of magnitude higher than those measured in carbon-doped single crystals ${ }^{5}$ or in good undoped samples. ${ }^{17,11}$ The normal state resistivity of the $X$ $=0.17$ sample $[\rho(40 \mathrm{~K})=9000 \mu \mathrm{ohm} \mathrm{cm}$ was nearly a factor of 10 higher than that of the $X=0.0525$ sample $960 \mu \mathrm{ohm} \mathrm{cm}]$. Such values are indicative of reduced connectivity, ${ }^{18}$ a conclusion supported by their essentially metallic temperature dependence and residual resistivity ratio (RRR) values (1.185 for $X=0.0525$ and 1.037 for $X$ $=0.17)$. It is interesting that the $J_{c}(H, T)$ values can still approach $10^{6} \mathrm{~A} / \mathrm{cm}^{2}$, even with this high resistivity. Like some SiC-doped wires of Dou et al. ${ }^{8}$ with high $\rho(40 \mathrm{~K})$ values, it appears that the inferred poor connectivity of the samples is not a barrier to attaining high $J_{c}$ values. An additional intrinsic source of high resistivities in both samples is likely due to incomplete recovery of milling-induced disorder during heat treatment, as supported by the weak XRD patterns with broad peaks. Such inhibited recovery is generally not observed in undoped samples, leading to the conclusion that the presence of carbon hinders eliminating defects after milling. The low $J_{C}$ of the $X=0.17$ sample and the conclusion that not all of the $\mathrm{C}$ is incorporated into the lattice indicates addi-

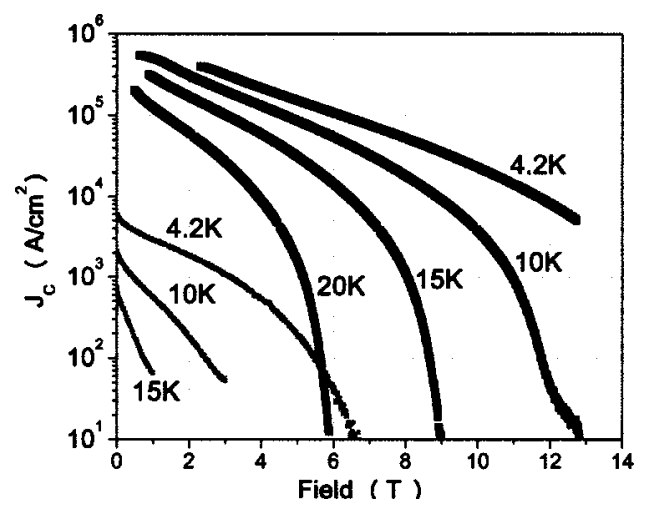

FIG. 3. Magnetization $J_{c}(H, T)$ values derived for $X=0.0525$ (thick lines) and $X=0.17$ (narrow lines). Comparison of the fields at which $J_{c}$ tends to

zero are about half the values of $H_{c 2}(T)$ found in Fig. 1 . 
tional obstruction by residual $\mathrm{C}$ phases, as has been seen in some C-doped films. ${ }^{19}$

In summary we have shown that milling $\mathrm{C}$ with $\mathrm{MgB}_{2}$ can produce $H_{c 2}(0 \mathrm{~K})$ equal to that obtained for single crystals and CVD filaments. Lattice disorder introduced in the milling process is indicated by weakened XRD patterns, high normal state resistivity, and a low-temperature upturn in $H_{c 2}(T)$.

B.J.S. acknowledges the support of the Fusion Energy Sciences Fellowship Program, administered by Oak Ridge Institute for Science and Education under a contract between the U.S. Department of Energy and the Oak Ridge Associated Universities. This research was also supported by the NSF under the University of Wisconsin-Madison MRSEC program. The authors thank the excellent staff of the NHMFL, Los Alamos, particularly Dr. F. Balakirev, as well as W. Starch, A. Squitieri, J. Mantei, and R. Mungall in Wisconsin.

${ }^{1}$ V. Braccini, A. Gurevich, J. E. Giencke, M. C. Jewell, C. B. Eom, D. C. Larbalestier, A. Pogrebnyakov, Y. Cui, B. T. Liu, Y. F. Hu, J. M. Redwing, Qi Li, X. X. Xi, R. K. Singh, R. Gandikota, J. Kim, B. Wilkens, N. Newman, J. Rowell, B. Moeckly, V. Ferrando, C. Tarantini, D. Marre, M. Putti, C. Ferdeghini, R. Vaglio, and E. Haanappel, Phys. Rev. B 71, 012504 (2005).

${ }^{2}$ A. Gurevich, Phys. Rev. B 67, 184515 (2003).

${ }^{3}$ T. Masui, S. Lee, A. Yamamoto, H. Uchiyama, and S. Tajima, Physica C 412-414, 303 (2004).

${ }^{4}$ E. Ohmichi, T. Masui, S. Lee, S. Tajima, and T. Osada, J. Phys. Soc. Jpn.
73, 2065 (2004)

${ }^{5}$ S. M. Kazakov, R. Puzniak, K. Rogacki, A. V. Mironov, N. D. Zhigadlo, J. Jun, Ch. Soltmann, B. Batlogg, and J. Karpinski, Phys. Rev. B 71, 024533 (2005).

${ }^{6}$ R. H. T. Wilke, S. L. Bud'ko, P. C. Canfield, D. K. Finnemore, R. J. Suplinskas, and S. T. Hannahs, Phys. Rev. Lett. 92, 217003 (2004).

${ }^{7}$ S. X. Dou, S. Soltanian, J. Horvat, X. L. Wang, S. H. Zhou, M. Ionescu, H. K. Liu, P. Munroe, and M. Tomsic, Appl. Phys. Lett. 81, 3419 (2002).

${ }^{8}$ S. X. Dou, V. Braccini, S. Soltanian, R. Klie, Y. Zhu, S. Li, X. L. Wang, and D. Larbalestier, J. Appl. Phys. 96, 1549 (2004).

${ }^{9}$ A. Matsumoto, H. Kumakura, H. Kitaguchi, and H. Hatakeyama, Supercond. Sci. Technol. 17, 319 (2004).

${ }^{10}$ M. D. Sumption, M. Bhatia, E. W. Collings, M. Rindfleisch, M. Tomsic, S. Soltanian, S. X. Dou, and E. W. Collings, Appl. Phys. Lett. 86, 092507 (2005).

${ }^{11}$ V. Braccini, L. D. Cooley, S. Patnaik, D. C. Larbalestier, P. Manfrinetti, A. Palenzona, and A. S. Siri, Appl. Phys. Lett. 81, 4577 (2002).

${ }^{12}$ R. Puzniak, M. Angst, A. Szewczyk, J. Jun, S. M. Kazakov, and J. Karpinski, e-print cond-mat/0404579.

${ }^{13}$ T. Takenobu, T. Ito, D. H. Chi, K. Prassides, and Y. Iwasa, Phys. Rev. B 64, 134513 (2001).

${ }^{14}$ A. Bharathi, S. J. Balaselvi, S. Kalavathi, G. L. N. Reddy, V. S. Sastry, Y. Hariharan, and T. S. Radhakrishnan, Physica C 370, 211 (2002).

${ }^{15}$ S. Lee, T. Masui, A. Yamamoto, H. Uchiyama, and S. Tajima, Physica C 397, 7 (2003).

${ }^{16}$ A. Serquis, L. Civale, D. L. Hammon, X. Z. Liao, J. Y. Coulter, Y. T. Zhu, M. Jaime, D. E. Peterson, F. M. Mueller, V. F. Nesterenko, and Y. Gu, Appl. Phys. Lett. 82, 2847 (2003).

${ }^{17}$ P. C. Canfield, D. K. Finnemore, S. L. Bud'ko, J. E. Ostenson, G. Lapertot, C. E. Cunningham, and C. Petrovic, Phys. Rev. Lett. 86, 2423 (2001).

${ }^{18}$ J. M. Rowell, Supercond. Sci. Technol. 16, 17 (2003).

${ }^{19}$ A. V. Pogrebnyakov, X. X. Xi, J. M. Redwing, V. Vaithyanathan, D. G. Schlom, A. Soukiassian, S. B. Mi, C. L. Jia, J. E. Giencke, C. B. Eom, J. Chen, Y. F. Hu, Y. Cui, and Qi Li, Appl. Phys. Lett. 85, 2017 (2004). 